\title{
Effects of Neoadjuvant Intraperitoneal/Systemic Chemotherapy (Bidirectional Chemotherapy) for the Treatment of Patients with Peritoneal Metastasis from Gastric Cancer
}

\author{
Yutaka Yonemura, ${ }^{1,2,3,4}$ Ayman Elnemr, ${ }^{1,5}$ Yoshio Endou, ${ }^{6}$ Haruaki Ishibashi, ${ }^{1,3}$ Akiyoshi \\ Mizumoto, ${ }^{2}$ Masahiro Miura, ${ }^{7}$ and ${\text { Yan } \mathrm{Li}^{8}}^{8}$ \\ ${ }^{1}$ NPO Organization to Support Peritoneal Surface Malignancy Treatment, Osaka, Kishiwada 596-0032, Japan \\ ${ }^{2}$ Department of Surgery, Kusatsu General Hospital, Shiga, Kusatsu 525-8585, Japan \\ ${ }^{3}$ Department of Surgery, Peritoneal Surface Malignancy Center, Kishiwada Tokushukai Hospital, Kishiwada 596-8522, Japan \\ ${ }^{4}$ Peritoneal Dissemination Program, Kishiwada Tokushukai Hospital and Kusatsu General Hospital, NPO Organization to Support \\ Peritoneal Surface Malignancy Treatment, 1-26, Haruki-Moto-Machi, Osaka, Kishiwada City, 596-0032, Japan \\ ${ }^{5}$ Department of Surgery, Tanta University Hospital, Tanta, Egypt \\ ${ }^{6}$ Department of Experimental Therapeutics, Cancer Research Institute, Kanazawa University, Kanazawa 920-1192, Japan \\ ${ }^{7}$ Department of Anatomy, School of Medicine, Oita University, Oita 870-1192, Japan \\ ${ }^{8}$ Department of Oncology, Zhongnan Hospital, Cancer Center of Wuhan University, Wuhan 430072, China
}

Correspondence should be addressed to Yutaka Yonemura, y.yonemura@coda.ocn.ne.jp

Received 13 January 2012; Accepted 29 April 2012

Academic Editor: Lapo Bencini

Copyright (c) 2012 Yutaka Yonemura et al. This is an open access article distributed under the Creative Commons Attribution License, which permits unrestricted use, distribution, and reproduction in any medium, provided the original work is properly cited.

\begin{abstract}
Novel multidisciplinary treatment combined with neoadjuvant intraperitoneal-systemic chemotherapy protocol (NIPS) and peritonectomy was developed. Ninety-six patients were enrolled. Peritoneal wash cytology was performed before and after NIPS through a port system. Patients were treated with $60 \mathrm{mg} / \mathrm{m}^{2}$ of oral S-1 for 21 days, followed by a 1-week rest. On days 1,8 , and $15,30 \mathrm{mg} / \mathrm{m}^{2}$ of Taxotere and $30 \mathrm{mg} / \mathrm{m}^{2}$ of cisplatin with $500 \mathrm{~mL}$ of saline were introduced through the port. NIPS is done 2 cycles before surgery. Three weeks after NIPS, 82 patients were eligible to intend cytoreductive surgery (CRS) by gastrectomy + D2 dissection + periotnectomy to achieve complete cytoreduction. Sixty-eight patients showed positice cytology before NIPS, and the positive cytology results became negative in 47 (69\%) patients after NIPS. Complete pathologic response on PC after NIPS was experienced in $30(36.8 \%)$ patients. Stage migration was experienced in 12 patients $(14.6 \%)$. Complete cytoreduction was achieved in 58 patients $(70.7 \%)$. By the multivariate analysis, complete cytoreduction and pathologic response became a significantly good survival. However the high morbidity and mortality, stringent patient selection is important. The best indications of the therapy are patients with good pathologic response and $\mathrm{PCI} \leq 6$, which are supposed to be removed completely by peritonectomy.
\end{abstract}

\section{Introduction}

In the past, peritoneal carcinomatosis (PC) from gastric cancer has been regarded as a terminal stage [1], and the most oncologists regarded as a condition only to be palliated. Preusser et al. published a response rate to chemotherapy of $50 \%$ of patients with stage IV gastric cancer, but the response rate was the lowest in patients with PC [2]. Ajani et al. reported that $\mathrm{PC}$ was the most common indication of failure of the intensive chemotherapy [3]. Accordingly, surgery alone or chemotherapy alone is not an adequate management for gastric cancer patients with PC.

Over the past two decades, a new multimodal treatment called cytoreductive surgery (CRS) [4] plus perioperative chemotherapy (POC) was proposed. POC includes neoadjuvant chemotherapy (NAC), hyperthermic intraperitoneal chemotherapy (HIPEC), and/or early postoperative intraperitoneal chemotherapy (EPIC), which takes the advantage of surgery to reduce the visible tumor burden and POC to eradicate peritoneal micrometastasis and 
peritoneal free cancer cells (PFCCs) [5]. Survival analyses after CRS plus HIPEC showed that complete cytoreduction is associated with survival improvement $[5,6]$. Neoadjuvant chemotherapy is proposed to reduce tumor burden before operation, resulting in the improvement of the incidence of complete cytoreduction [5]. A new bidirectional chemotherapy (neoadjuvant intraperitoneal-systemic chemotherapy protocol (NIPS)) was developed to induce a reduction of the peritoneal cancer index of PC and to eradicate PFCCs [5]. NIPS can attack PC from both sides of peritoneum, not only from the peritoneal cavity but also from the subperitoneal blood vessels. Accordingly, NIPS is called as bidirectional chemotherapy.

In the present study, the effects of NIPS on the intraperitoneal cytological status, histological response of PC, the incidence of complete cytoreduction, and survivals in the patients with established PC form gastric cancer will be reported.

\section{Patients and Methods}

2.1. Patients. Ninety-six patients with primary gastric cancer with PC were enrolled in the study. Enrolled patients in the study were treated between April 2004 and December 2011. PC was diagnosed by biopsy under laparotomy, laparoscopy, or by the cytologic examination of ascites. The eligibility criteria included (1) histologically or cytologically proven PC from gastric adenocarcinoma; (2) absence of hematogenous metastasis and remote lymph node metastasis; (3) age 75 years or younger; (4) Eastern Clinical Oncology Group scale of performance status 2 or less; (5) good bone marrow, liver, cardiac, and renal function; (6) absence of other severe medical conditions or synchronous malignancy.

Informed consent according to the institutional guideline was obtained from all patients.

2.2. Methods to Introduce a Peritoneal Port System and Peritoneal Wash Cytology. A peritoneal port system (Hickman subcutaneous port; Bard, Salt Lake City, USA) was introduced into the abdominal cavity under local anesthesia, and the tip of the system was placed on the cul-de-sac of Douglas. Then, a peritoneal wash cytology was performed after $500 \mathrm{~mL}$ of physiological saline had been injected into the peritoneal cavity. To improve the accuracy of the cytology, an immunohistochemical examination using monoclonal antibodies for anti-human carcinoembryonic antigen (TAKARA Bio INC., Tokyo, Japan) and anti-human epithelial antigen (DAKO, Copenhagen, Denmark) were performed. A peritoneal wash cytological examination was performed before and after NIPS.

2.3. Bidirectional Chemotherapy. Patients were treated with $60 \mathrm{mg} / \mathrm{m}^{2}$ of oral S-1 (Taiho Pharmaceutical Co., Ltd., Tokyo, Japan) for 21 days, followed by a 1-week rest. On days 1,8 , and 15 after the start of oral S-1 administration, $30 \mathrm{mg} / \mathrm{m}^{2}$ of Taxotere and $30 \mathrm{mg} / \mathrm{m}^{2}$ of cisplatinum with $500 \mathrm{~mL}$ of saline were introduced through the port. This regimen was repeated after a one-week rest [7]. Bidirectional chemotherapy is done 2 cycles before surgery. The aims of NIPS are to reduce the peritoneal surface involved by PC and to eradicate PFCCs. Toxicities were graded using the CTCAE v 3.0.

2.4. Selection Criteria of Patients for Cytoreductive Surgery (CRS) after NIPS. After two cycles of NIPS, patients who had the following criteria are excluded as the candidates for CRS: (1) evidence of para-aortic lymph node involvement and distant hematogenous metastasis confirmed by computed tomography (CT), or magnetic resonance imaging (MRI), (2) patients with progressive disease after NIPS, or (3) patients with severe comorbidities or poor general condition.

2.5. Quantitative Evaluation of the Volume of PC and Assessment Completeness of Cytoreduction. Intraoperatively, the tumor volume was quantified according to the Japanese general rules for gastric cancer study [8] and the Sugarbaker's peritoneal cancer index (PCI) [9]. The abdomen and pelvis were divided into nine regions and the small bowel into four each assigned a lesion size (LS) score of $0-3$, representative of the largest implant visualized. LS- 0 denotes the absence of implants, LS- 1 indicates implants $0.25 \mathrm{~cm}$, LS-2 implants between 0.25 and $5 \mathrm{~cm}$, and LS-3 implants $>5 \mathrm{~cm}$ or a confluence of disease. These figures amount to a final numerical score of 0-39 (Figure 1).

The aim of CRS was to obtain complete macroscopic cytoreduction as a precondition for the application of HIPEC. The residual disease was classified intraoperatively using the completeness of cytoreduction (CC) score [9]. CC-0 indicates complete cytoreduction with no residual macroscopic nodule; $\mathrm{CC}-1$ indicates no macroscopic tumor but a positive histological margin on the esophageal, duodenal stump, or suspicious residual nodules less than $5 \mathrm{~mm}$ in diameter, CC-2 indicates apparent macroscopic residual tumors greater than $5 \mathrm{~mm}$ but upto $5 \mathrm{~cm}$ in diameter, and CC-3 indicates residual PC greater than $5 \mathrm{~cm}$ in diameter.

2.6. Methods of CRS Using Peritonectomy Techniques [6]. Laparotomy was done 3 weeks after the last day of NIPS. Under general anesthesia, midline incision was made from the xiphoid to the pubis. Just after laparotomy, peritoneal wash cytology is done, and PCI score was calculated in each case.

For the tissue dissection, electrosurgical techniques are used. In electrosurgery, a generator delivers high-frequency current greater than $200 \mathrm{kHz}$ under high-power electricity (100 Watt), using the electrosurgical generator (Valleylab Inc., Boulder, CO, USA). The mainly used handpiece is the ball-tipped type. The $2 \mathrm{~mm}$ ball-tip electrode is used for dissecting on visceral surfaces.

After the left lobe of liver is freed from the left triangular ligament, resection of the lesser omentum along the Arantius duct is started. Gastrectomy combined with D2 dissection [8], greater omentectomy, splenectomy, and the resection of anterior leaf of mesocolon is done. Importantly, the small bowel should be intact for the safe reconstruction either by esophagojejunostomy or gasrojejunostomy. The aim of 


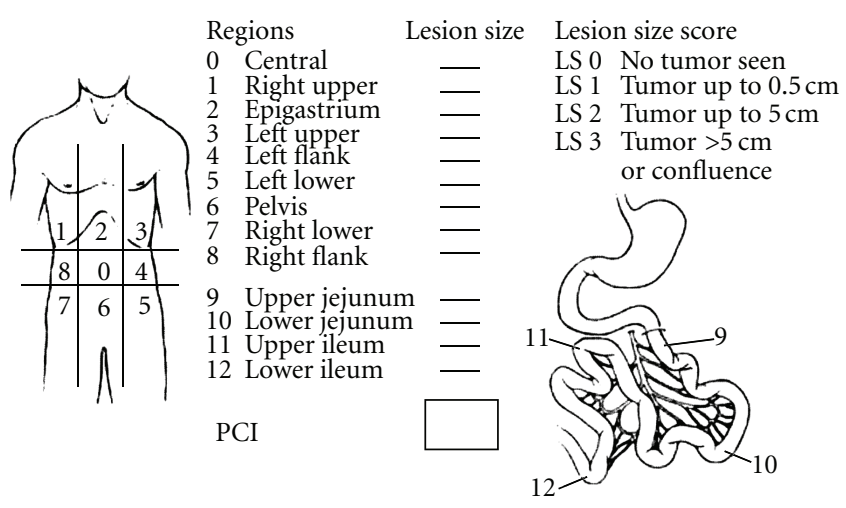

FIgURE 1: Peritoneal cancer index (PCI). Peritoneal cavity is divided into 13 parts, which ranges from 0 to 12 . Accurate measurement of each region is scored as lesion size 0 through 3. LS 0: no implants.

peritonectomy is to remove all the macroscopic PC nodules with peritoneum. If the parietal peritoneum is involved, both sides of the parietal peritoneum are peeled off from the posterior rectus sheath to the retroperitoneal space. The dissection continues deeply and in a counterclockwise direction, starting in the right flank till reaching the peritoneum covering the left copula of the diaphragm. Then, the dissection is completed in the upper right side till reaching the anterior renal fascia, inferior vena cava, and posterior wall of the duodenum.

The peritoneum of the Morrison's pouch and paracolic gutters on both sides are completely freed from retroperitoneum and is removed with the anterior parietal peritoneum.

If the undersurface of the diaphragm is involved, stripping of peritoneum from the right and left hemidiaphragm is done. The falciform and round ligament are taken down and resected completely.

Large PC nodules attach on the transverse colon are removed in combination with extended right hemicolectomy.

The entire pelvic peritoneum is dissected from the anterior inferior abdominal wall, urinary bladder, and retroperitoneum. The peritoneum covering the urinary bladder is dissected and the rectovesical pouch is completely freed from the urinary bladder and rectum. In male, the space between seminal vesicle and peritoneum of rectovesical pouch is dissected, lifting the vas deferens off. In female, blood vessels around the uterus are dissected and cut with LigaSure (Valleylab Inc., Boulder, CO, USA). Amputation of vagina is done at a plane $1 \mathrm{~cm}$ below the peritoneal reflection of Douglas pouch to ensure removal of all tumor occupy the cul-de-sac.

If the tumor invades into the anterior rectal wall, rectum is cut at $1 \mathrm{~cm}$ below the peritoneal reflection. Reasonable length of the rectum should be preserved for the anastomosis with the colon.

The entire small bowel and its mesentery are traced from the duodenojejunal flexure to the ileocecal junction. Then, both sides of the mesentery are inspected and palpated, and the tumor nodules excised with electrosurgery. Complete cytoreduction is aimed by removing all macroscopic tumors by peritonectomy combined with electric fulguration.

2.7. Histologic Evaluation of NIPS. Histologic effects on primary tumors and PC were evaluated according to the general rules for gastric cancer treatment [8]. Histological response after chemotherapy is classified into 4 categories. Ef- 0 shows no histologic response or response less than onethird of the tumor tissue. A histologic Ef-1 means that the degeneration of cancer is detected in the tumor tissue raging from one-third to less than two-thirds of the tumor tissue. EF-2 shows the degeneration of cancer tissue in wider than two-thirds of the tumor tissue, while an Ef-3 means the complete disappearance of the cancer cells.

2.8. Statistical Analyses. All patients were followed and no patients were lost to follow up. Outcome data were obtained from medical records and patients' interview. All statistical analyses had performed using SPSS software statistical computer package version 17 (SPSS Inc., Chicago, USA).

\section{Results}

Clinical characteristics of the 96 patients are listed in Table 1.

The average age was 51.3 years, including 42 men and 54 women. All 96 patients had primary gastric cancer and had P2 or P3 dissemination. Ascites was found in 55 (57\%) patients.

Before NIPS, cytology had been positive in $68(70.8 \%)$ of 96 patients and was positive in $21(22.9 \%)$ after NIPS. These 68 positive cytology results before NIPS became negative in $47(69 \%)$ patients after NIPS (Table 2).

After NIPS, 82 patients received operation, and the other 14 patients did not undergo operation due to the progression of disease or refusal of operation. At laparotomy, $\mathrm{P}$ status in Japanese rules became to be $\mathrm{P} 0$ in $7, \mathrm{P} 1$ in $11, \mathrm{P} 2$ in 8 , and $\mathrm{P} 3$ in 56 patients. Mean PCI was 6.3, ranging from 0 to 33, and $\mathrm{PCI} \leq 6$ and $\mathrm{PCI} \geq 7$ were 56 and 26 patients, respectively.

Table 3 indicates the operation methods. Total gastrectomy was performed in 67 patients. A variety of supplemental procedures were performed to achieve tumor cytoreduction. The common procedures for visceral peritonectomy were transverse colectomy combined with right hemicolectomy and omentectomy $(N=33)$, pelvic peritonectomy in 38 including low anterior resection in 17, bilateral salpingooophorectomy in 36 of 48 female patients, segmental resection of small bowel, and small-bowel mesentery in 18 and 16 patients. Left and right subdiaphragmatic peritonectomy was performed in 25 and 22 patients, respectively. Mean operation time was $230 \mathrm{~min}(120 \sim 690 \mathrm{~min})$, and mean blood loss was $1571 \mathrm{~mL}(850 \sim 4540 \mathrm{~mL})$.

Complete cytoreduction (CC- 0 ) was achieved in 58 of 82 patients $(70.7 \%)$. CC-0 was achieved in $48(78.7 \%)$ of 61 patients with negative cytological status after NIPS, but was done in $10(47.6 \%)$ of 21 patients with positive cytology after NIPS. Regarding the PCI, CC-0 was done in 54 (96.4\%) of 56 patients with PCI score $\leq 6$, but was performed in 
TABLE 1: Clinicopathologic characteristics of 96 primary gastric cancer patients with PC.

\begin{tabular}{|c|c|c|c|}
\hline & Results & CRS $(N=82)$ & No operation $(N=14)$ \\
\hline Age, years (median) & $25-76(51.3)$ & $25-74(52.2)$ & $28-75(46.9)$ \\
\hline Gender (male/female) & $42 / 54$ & $34 / 48$ & $8 / 6$ \\
\hline \multicolumn{4}{|l|}{ Histologic type } \\
\hline Differentiated & & 3 & 1 \\
\hline Poorly differentiated & & 79 & 13 \\
\hline \multicolumn{4}{|l|}{ Lymph node metastasis } \\
\hline pNo & & 16 & \\
\hline pN1 & & 40 & \\
\hline $\mathrm{pN} 2$ & & 17 & \\
\hline $\mathrm{pN} 3$ & & 9 & \\
\hline \multicolumn{4}{|l|}{ Macroscopic type } \\
\hline Type 3 & & 16 & 3 \\
\hline Type 4 & & 66 & 11 \\
\hline Liver metastasis & 0 & 0 & 0 \\
\hline \multicolumn{4}{|l|}{ Completeness of cytoreduction } \\
\hline Complete cytoreduction (CC-0) & & 61 & \\
\hline Incomplete cytoreduction (CC-1 3) & & 21 & \\
\hline \multicolumn{4}{|c|}{ Hyperthermic intraoperative chemotherapy (HIPEC) } \\
\hline Done & & 53 & \\
\hline Not done & 29 & & \\
\hline
\end{tabular}

TABLE 2: Changes of peritoneal lavage cytology before and after NIPS.

\begin{tabular}{lccc}
\hline Wash cytology after NIPS & & & \\
Wash cytology before NIPS & Negative & Positive & Total \\
\hline Negative & 27 & 1 & 28 \\
Positive & 47 & 21 & $68(70.8 \%)$ \\
\hline 74 & $22(22.9 \%)$ & 96 & \\
\hline
\end{tabular}

TABLE 3: Surgical procedures for CRS.

\begin{tabular}{lc}
\hline Surgical procedures & Patients number \\
\hline Gastrectomy & 67 \\
Total gastrectomy & 15 \\
$\quad$ Subtotal distal gastrectomy & 22 \\
Resection of right diaphragmatic copula & 25 \\
Resection of left diaphragmatic copula & 82 \\
Greater omentectomy & 38 \\
Pelvic peritonectomy & $34 / 48$ \\
Hysterectomy & $36 / 48$ \\
Salpingo-oophorectomy & 33 \\
Right hemicolectomy & 17 \\
Low anterior resection & 18 \\
Small-bowel resection & 16 \\
Resection of small-bowel mesentery &
\end{tabular}

$10(38.4 \%)$ of 26 patients with PCI $\geq 7$. Causes of incomplete cytoreduction were diffuse involvement of small bowel in 7 patients, PCI score higher than 20 in 6 patients, positive margin on esophageal stump in 3 patients, and local invasion into the retroperitoneal tissue in one patient.
TABLE 4: Adverse effects after NIPS.

\begin{tabular}{lccc}
\hline Side effects & Grade 3 & Grade 4 & Grade 5 \\
\hline $\begin{array}{l}\text { Hematological } \\
\text { Leukopenia }\end{array}$ & $1(1.0 \%)$ & 0 & 0 \\
$\quad \begin{array}{l}\text { Thrombocytopenia } \\
\text { Nonhematological }\end{array}$ & $1(1.0 \%)$ & $1(1.0 \%)$ & 0 \\
$\quad$ Stomatitis & 0 & 0 & 0 \\
$\quad \begin{array}{l}\text { Diarrhea } \\
\text { Nausea, vomiting }\end{array}$ & $1(1.0 \%)$ & 0 & 0 \\
$\quad$ Fatigue & $1(1.0 \%)$ & 0 & $1(1.0 \%)$ \\
$\quad$ Renal function (need & $2(2.1 \%)$ & $1(1.0 \%)$ & 0 \\
hemodialysis) & $1(1.0 \%)$ & 0 & 0 \\
\hline 7 (7.3\%) & $2(2.1 \%)$ & $1(1.0 \%)$ & \\
\hline
\end{tabular}

During NIPS, side effects of level 3, 4, and 5 were found in $7(7.3 \%), 2(2.1 \%)$, and $1(1.0 \%)$ patients (Table 4$)$. One patients died of aspiration pneumonia due to ileus (grade 5).

After NIPS and CRS, 8, 9, and 3 patients developed grade 3,4 , and 5 complications (Table 5). The most frequent complications are anastomosis dehiscence. The overall operative mortality rate was $3.7 \%(3 / 82)$, and the cause of 
death was multiple organ failure with renal failure, hepatic coma, and sepsis due to anastomosis leakage. Grade 4 complications were found in 9 patients, and three patients developed renal failure were treated by hemodialisis. Six patients underwent operation for the postoperative bleeding in one patient, drainage of the abscess from anastomotic leakage in four patients, and port infection in one patient. All 8 patients developed grade 3 complications recovered well after appropriate treatments.

Twenty-six (31.7\%) were alive at the time of analysis. The survival curve for all patients is shown in Figure 2. Median survival time (MST) of patients who underwent CRS was 14.4 months, with a one-, three-, and five-year survival of $61 \%, 16 \%$, and $16 \%$, respectively. MST of patients who did not receive operation was 9.0 months (Figure 2). There was a significant survival difference between the two groups $(P<$ $0.05)$. Patients who received a complete resection (CC- 0 ) had an MST of 21.1 months, and MST of patients who received incomplete cytoreduction $(\mathrm{CC}-1 \sim 3)$ was 8.4 months $(P<$ 0.001 ) (Figure 3 ). Significant survival difference was found between CC- 0 and CC- $1 \sim 3$ group. MST of patients with PCI $\leq 6$ was 20.4 months with a 5 -year survival of $21.0 \%$ and that of patients with PCI $\geq 7$ was 9.6 months with no 5-year survival. There was a significant survival difference between the two groups $(P<0.001)$ (Figure 4$)$.

Histologic effects on primary tumors were found in 71 of the 82 tumors, and Ef- $1,-2,-3$ response in the primary tumors were detected in $43(52.5 \%), 28(34.1 \%)$, and 0 tumor $(0 \%)$, respectively (Table 6). In contrast, the complete histologic disappearance of PC was observed in 30 (36.8\%) of 82 patients (Table 6). Stage migration from stage 4 to stage 1,2 , or 3 was experienced in 2,2 , and 8 patients. MST of patients with Ef-0/Ef- 1 effects in PC tissue was 5.8 months with a 5 -year survival of $0 \%$ and that of patients with Ef-2/Ef-3 effects was 24.0months with a 5-year survival of $28.0 \%$ (Figure 5). There was a significant survival difference between the two groups $(P<0.001)$.

As shown in Table 7, among various prognostic factors, CC score and pathologic effects were independent prognostic factors.

Recurrence was found in peritoneum, lung, liver hilum, liver, and bone in $27,5,3,2$, and 2 patients, respectively.

\section{Discussion}

The current state-of-the-art treatment for colorectal peritoneal dissemination consists of a comprehensive management strategy using CRS and POC [10]. Patients with a low tumor volume, well/moderately differentiated tumors, and complete cytoreduction may potentially benefit from combined treatment. In gastric cancer patients with PC, no survival benefit has been reported by cytoreduction alone [1]. In contrast, CRS with peritonectomy plus HIPEC confers a prolonged survival period [5]. Furthermore, complete cytoreduction is an essential factor for a good outcome, and NIPS plus peritonectomy may improve the incidence of complete cytoreduction [7]. The aims of neoadjuvant chemotherapy (NAC) are stage reduction, eradication of

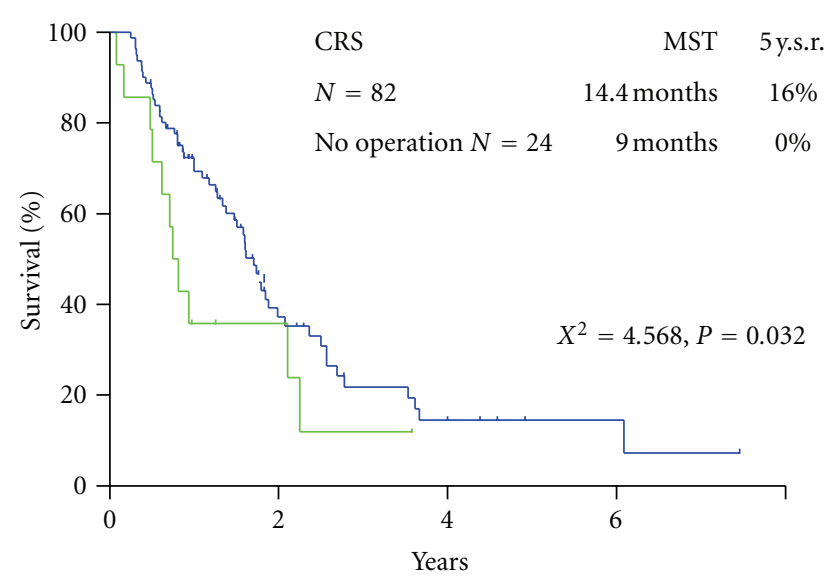

FIgURE 2: Survivals of 82 patients who underwent CRS, and 14 patients who did not underwent CRS.

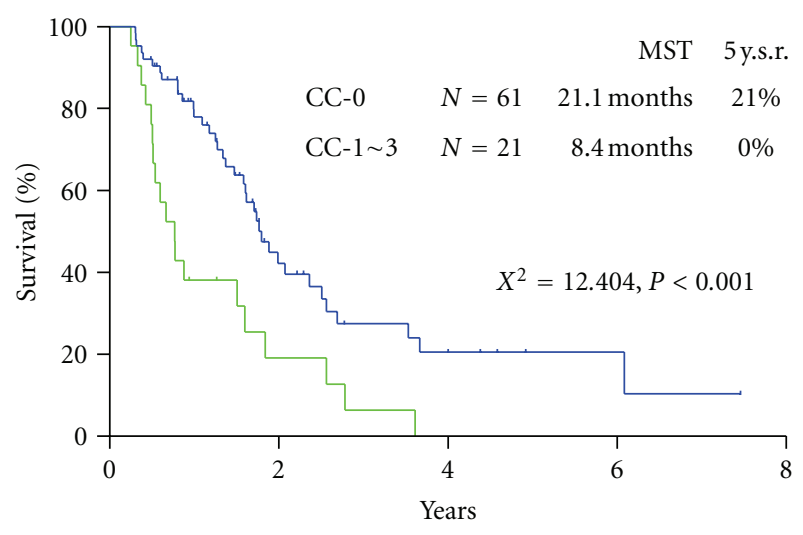

FIgURE 3: Survival difference after CC-0 and CC-1 resection.

micrometastasis outside the surgical field, and the improvement of resectability. Systemic chemotherapy usually is used for NAC. In the late 1990s, TS-1, irinotecan, taxanes, and docetaxel were introduced for gastric cancer treatment, and the response rates after monotherapy with these drugs were around 20\%. Combination chemotherapy with S-1 and CDDP produced outstanding results, with a response rate of $74 \%$ [11]. Yabusaki et al. reported the results of NAC with S1 and CDDP in 37 advanced gastric cancer patients. After 2 courses of treatment, the overall response rate was $68 \%$, but the response rate for patients with peritoneal dissemination was only $14 \%$ (2/14) [12]. S-1 plus CPT-11 and CPT-11 plus CDDP produced a high-response rate of $42 \%$ and a long period of progression-free survival, but treatment failure as a result of toxicity was also observed [13].

These results indicated that systemic chemotherapies have minimal effects on PC. In other words, the peritoneal cavity acts as a sanctuary against systemic chemotherapy, probably because of the existence of a blood-peritoneal barrier consisting of stromal tissue between mesothelial cells and submesothelial blood capillaries $[9,14]$. This barrier accounts for a total thickness of $90 \mu \mathrm{m}$ [14]. Accordingly, only a small amount of systemic drugs are capable of 
TABLE 5: Complications after NIPS and CRS.

\begin{tabular}{lccc}
\hline & & Complications & \\
Grade $1-2$ & Grade 3 & Grade 4 & Grade 5 \\
$N=4(4.9 \%)$ & $N=8(9.8 \%)$ & Renal failure: 3 & MOF from leakage: 3 \\
\hline Minor leakage: 2 & Pancreas fistula: 2 & Major leakage: 4 & \\
& Major leakage: 4 & Bleeding: 1 & \\
Abd. wall dehiscence: 1 & Abdominal bleeding: 1 & & \\
Abdominal abscess: 1 & Port infection: 1 & & \\
\hline
\end{tabular}

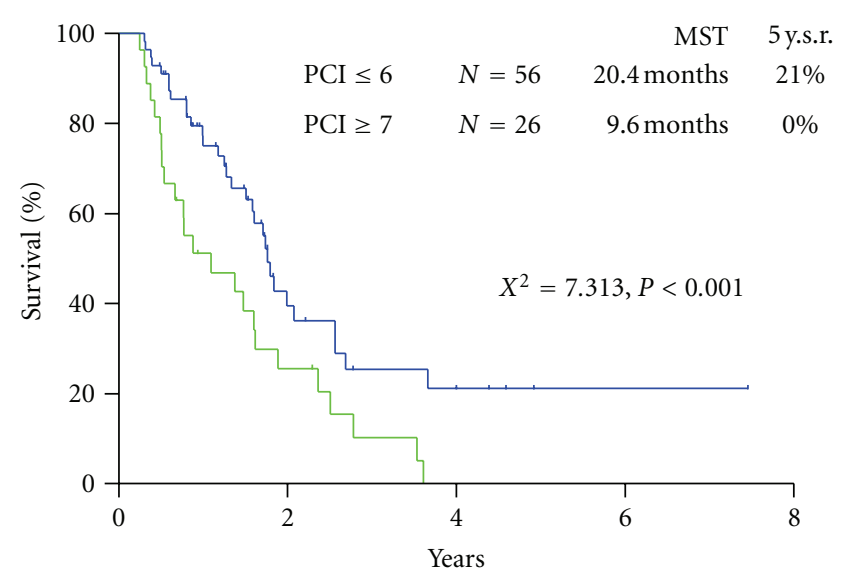

Figure 4: Survival difference of patients with PCI $\leq 6$ and those with $\mathrm{PCI} \geq 7$.

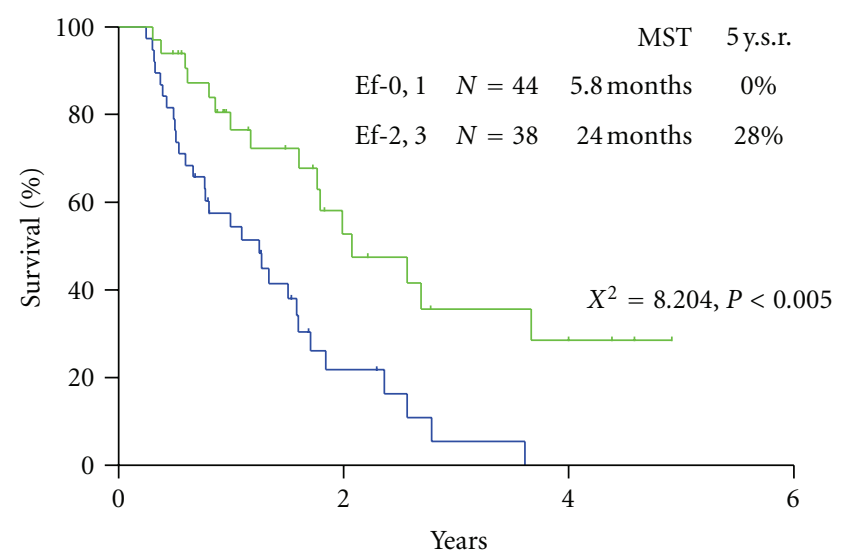

FIGURE 5: Survival difference of patients, according to the histological response on PC after NIPS.

TABLE 6: Pathological response after NIPS.

\begin{tabular}{lcccc}
\hline \multicolumn{5}{c}{ Responder } \\
Ef-0 & Ef-1 & Ef-2 & Ef-3 \\
\hline Primary tumor $11(13.4 \%)$ & $43(52.5 \%)$ & $28(34.1 \%)$ & $0(0 \%)$ \\
PC & $25(30.4 \%)$ & $20(24.3 \%)$ & $7(8.5 \%)$ & $30(36.8 \%)$ \\
\hline
\end{tabular}

penetrating this barrier and passing into the peritoneal cavity, and a higher percentage of the administered drugs instead moves to the bone marrow and vital organs other than the peritoneum, resulting in the development of adverse effects.

In contrast, IP chemotherapy offers potential therapeutic advantages over systemic chemotherapy by generating high local concentrations of chemotherapeutic drugs in the peritoneal cavity $[15,16]$. This advantage of IP chemotherapy can be expressed by the area under the curve (AUC) ratios of intraperitoneal versus plasma exposure.

Relatively high AUC IP/IV ratios were obtained after the IP administration of paclitaxel, docetaxel, gemcitabine, 5fluorouracil, and doxorubicin [16]. These drugs may be good candidates for IP chemotherapy.

Other important factors in the selection of drugs for IP chemotherapy are a high-penetration activity into the PC nodules and chemosensitivity. Cisplatin and carboplatin have the highest penetration activity and were confirmed to penetrate 1 to $2 \mathrm{~mm}$ from the surface of PC nodules [15]. In an experimental PC model using a highly metastatic cell line derived from human gastric cancer, docetaxel, 5-FU, carboplatin, and TS-1 plus cisplatin was highly effective for improving the survival of nude mice bearing PC, and the IP administration of these drugs is expected to become a standard therapy for gastric cancer patients $[17,18]$.

From these clinical and experimental results, a new bidirectional chemotherapy combined with the oral administration of S-1 and IP CDDP and docetaxel has been developed. By simultaneously administering intravenous and intraperitoneal chemotherapy, a bidirectional diffusion gradient can create a wider treatment area than single treatment.

The Cox multivariate analysis clearly demonstrated the complete cytoreduction and pathological response after NIPS were the independent prognostic factors. The factors to achieve CC-0 resection correlated with the negative cytology after NIPS and PCI score $\leq 6$. Furthermore, peritonectomy techniques enable to achieve CC-0 resection even in the patients with higher PCI score $[6,7]$.

Preoperative evaluation of $\mathrm{PC}$ from gastric cancer is limited, and the sensitivity of CT for detecting PC was influenced by the lesion size. Koh et al. reported the value of preoperative CT in estimating PC in patients with colorectal carcinomatosis [19]. The depiction rate of small-bowel involvement had the lowest sensitivity, with a rate of $8-17 \%$., and the false-negative rate significantly decreased with the lesion size. Small nodules $(<0.5 \mathrm{~cm})$ were visualized using CT with a sensitivity of $11 \%$, in contrast to a sensitivity of $94 \%$ for PC with a diameter greater than $5 \mathrm{~cm}$. Accordingly, preoperative assessment of PCI by CT is not recommended in gastric cancer. In contrast, cytological examination from a port 
TABLE 7: Prognostic parameters (results of Cox proportional hazard model and logrank test).

\begin{tabular}{lcccccc}
\hline & \multicolumn{2}{c}{ Cox hazard model } & \multicolumn{2}{c}{ Logrank test } & \\
$X^{2}$ & $P$ & Realtive sisk & $95 \%$ CI & $X^{2}$ & $P$ & \\
\hline Sex (male versus female) & 2.158 & 0.141 & 0.652 & $0.3697-1.1531$ & 4.298 & 0.038 \\
Age $(\leq 65$ versus $>65)$ & 0.603 & 0.437 & 1.399 & $0.5991-3.2692$ & 0.289 & 0.59 \\
Histologic type (diff. versus poorly) & 0.024 & 0.876 & 1.171 & $0.1584-8.6651$ & 0.631 & 0.427 \\
CC (CC-0 versus CC-1) & 4.197 & 0.04 & 2.005 & $1.0306-3.9041$ & 8.537 & 0.003 \\
PCI $(\geq 6$ versus $\leq 7)$ & 1.592 & 0.206 & 1.528 & $0.7908-2.9536$ & 5.737 & 0.017 \\
Pathologic response on PC (Ef-1, -2 versus Ef-2, -3) & 4.269 & 0.038 & 0.429 & $0.1927-0.9575$ & 10.303 & 0.001 \\
LN status (pN0 versus pN1, 2, and 3) & 2.478 & 0.115 & 2.121 & $0.8317-5.4108$ & 3.739 & 0.053 \\
Cytology after NIPS (class I versus class V) & 0.047 & 0.828 & 1.079 & $0.5415-2.1513$ & 0.365 & 0.546 \\
\hline
\end{tabular}

system is very convenient and is an objective evaluation of the intraperitoneal cytologic status. In the present study, CC0 was achieved in $48(78.7 \%)$ of 61 patients with negative cytological status after NIPS, but was done in 10 (47.6\%) of 21 patients with positive cytology after NIPS. There was a significant difference in the incidence of CC-0 resections between the two groups. Accordingly, cytologic examination through a port system may be one of the indicators for CC0 resection. Furthermore, before NIPS, cytology had been positive in $68(70.8 \%)$ of 96 patients and was positive in 21 (22.9\%) after NIPS. These 68 positive cytology results before NIPS became negative in $47(69 \%)$ patients after NIPS. NIPS can eradicate PFCCs before CRS and may prevent the attachment of PFCCS on the surgical wound at CRS.

After systemic neoadjuvant chemotherapy, a complete PC response is very rare. Inokuchi et al. reported that the response rate of PC after S-1 plus irinotecan was $69 \% 9 / 13$ ), but no CR was experienced for PC [20]. Baba et al. also reported the limited effects of systemic S-1+CDDP on PC from gastric cancer [21].

After histological examination of the primary tumors and the resected peritoneum, histologic effects on primary tumors were found in 71 of the 82 tumors. However, the Ef- 2 response and the complete pathologic response of Ef3 in primary tumors were found in 28 tumors $(34.1 \%)$ and 0 tumor $(0 \%)$. In contrast, those in the peritoneal dissemination were observed in $30(36.8 \%)$ of 82 patients. In addition, stage migration from stage 4 to stage 1,2 , or 3 was experienced in 12 patients $(14.6 \%)$. These results indicate that NIPS can be a powerful strategy for eradicating PFCCs and for the reduction of the PCI score.

The present study demonstrated that the survival results were significantly better when the PCI was lower than 6. CC0 resection was done significantly higher in patients with PCI $\leq 6$ than $\mathrm{PCI} \geq 7$. The frequent cause of incomplete cytoreduction was the diffuse involvement of the small bowel. NIPS can reduce PCI score, and the timing of peritonectomy can be determined by the laparoscopic examination. Garofalo reported an excellent experience of laparoscopic diagnosis for PC [22]. There was a good correlation between the opensurgery data and the laparoscopic PCI scores. If the PCI score determined by the laparoscopy is larger than 7 accompanying with small-bowel involvement, NIPS is recommended to reduce PCI score on the small bowel. In contrast, patients with a PCI score $>7$ even after NIPS should be treated with palliative intent without peritonectomy. The PCI score is believed to be an independent prognostic factor, and a PCI score is capable of serving as a threshold for favorable versus poor prognosis.

NIPS may add to the morbidity and mortality of further surgical treatment [7]. The incidence of major side effects (grade 3, 4, and 5) after NIPS was 10.4\% (10/96). Chemotherapy-related death was found in one patient, and she died of aspiration pneumonia due to bowel obstruction without relation with chemotherapy. Renal dysfunction occurred in one patients (1.0\%), but the patients recovered fully by hemodialysis. Accordingly, the new bidirectional chemotherapy regimen is considered to be a safe method as compared with the results of previous reports of systemic chemotherapy $[2,3,23,24]$.

The present study demonstrated that NIPS plus peritonectomy may improve the incidence of complete cytoreduction. However, NIPS might increase the risk of a peritonectomy procedure plus a gastrectomy combined with a lymphadenectomy. Glehen reported a mean operation time of 5.2 hours (range, 1.5-9.5 hours), a 30-day mortality rate of $4 \%(2 / 49)$, and a major complication rate of $27 \%$ (13/49) [22]. In the present study, three hospital deaths $(3.7 \%)$ occurred in patients who died of multiple organ failure (MOF) from a pancreatic fistula, anastomotic leakage, and sepsis. Postoperative major complications occurred in $20(24.4 \%)$ patients. A second operation was necessary in 6 patients, who had complications from leakage of esophagojejunal anastomosis, bleeding, ileal and colonic fistulae, and port-site infection. Glehen reported a higher complication rate of $47 \%$ in patients who underwent extensive cytoreductive surgery (gastrectomy combined with the removal of more than 2 peritoneal zones) [22]. The magnitude of surgery, the number of resected organs, the number of anastomoses, and the operation time are considered to have contributed to the significantly higher complication rate. To avoid futile aggressive treatments, the stringent selection of patients must be emphasized preoperatively. Surgeons should have not only a large amount of surgical experiences with gastrointestinal and genitourinary diseases, but also an extensive knowledge of organ anatomy and physiology. Also, surgeons must be able to judge the balance between the postoperative risk associated with the magnitude of the peritonectomy and the survival benefit and quality of life after the aggressive treatment. 
In conclusion, NIPS and complete cytoreduction are the essential treatment modalities for the improvement of survival of patients with PC from gastric cancer. Surgeons should experience a learning curve with this procedure at the specialized center and should recommend the accumulation of experience to achieve an acceptable morbidity rate.

\section{References}

[1] B. Sadeghi, C. Arvieux, O. Glehen et al., "Peritoneal carcinomatosis from non-gynecologic malignancies: results of the EVOCAPE 1 multicentric prospective study," Cancer, vol. 88, no. 2, pp. 358-363, 2000.

[2] P. Preusser, H. Wilke, W. Achterrath et al., "Phase II study with the combination etoposide, doxorubicin, and cisplatin in advanced measurable gastric cancer," Journal of Clinical Oncology, vol. 7, no. 9, pp. 1310-1317, 1989.

[3] J. A. Ajani, D. M. Ota, J. M. Jessup et al., "Resectable gastric carcinoma: an evaluation of preoperative and postoperative chemotherapy," Cancer, vol. 68, no. 7, pp. 1501-1506, 1991.

[4] P. H. Sugarbaker, "Peritonectomy procedures," Annals of Surgery, vol. 221, no. 1, pp. 29-42, 1995.

[5] Y. Yonemura, E. Bandou, K. Kinoshita et al., "Effective therapy for peritoneal dissemination in gastric cancer," Surgical Oncology Clinics of North America, vol. 12, no. 3, pp. 635-648, 2003.

[6] P. H. Sugarbaker and Y. Yonemura, "Clinical pathway for the management of resectable gastric cancer with peritoneal seeding: best palliation with a ray of hope for cure," Oncology, vol. 58, no. 2, pp. 96-107, 2000.

[7] Y. Yonemura, Y. Endou, M. Shinbo et al., "Safety and efficacy of bidirectional chemotherapy for treatment of patients with peritoneal dissemination from gastric cancer: selection for cytoreductive surgery," Journal of Surgical Oncology, vol. 100, no. 4, pp. 311-316, 2009.

[8] Japanese Research Society for Gastric Cancer, The General Rules for Gastric Cancer Study, Kanehara Shuppan, Tokyo, Japan, 1st edition, 1995.

[9] T. S. Sugarbaker, D. Chang, P. Koslowe, and P. H. Sugarbaker, "Patterns of spread of recurrent intraabdominal sarcoma. Peritoneal carcinomatosis: 4," in Peritoneal Carcinomatosis4 Principles of Management, P. H. Sugarbaker, Ed., pp. 65-78, Kluwer Academic Publisher, 1996.

[10] V. J. Verwaal, S. Bruin, H. Boot, G. van Slooten, and H. van Tinteren, "8-year follow-up of randomized trial: cytoreduction and hyperthermic intraperitoneal chemotherapy versus systemic chemotherapy in patients with peritoneal carcinomatosis of colorectal cancer," Annals of Surgical Oncology, vol. 15, no. 9, pp. 2426-2432, 2008.

[11] W. Koizumi, S. Tanabe, K. Saigenji et al., "Phase I/II study of S-1 combined with cisplatin in patients with advanced gastric cancer," British Journal of Cancer, vol. 89, no. 12, pp. 22072212, 2003.

[12] H. Yabusaki, A. Nashimoto, and O. Tanaka, "Evaluation of TS1 combined with cisplatin for neoadjuvant chemotherapy in patients with advanced gastric cancer," Cancer \& Chemotherapy, vol. 30, no. 12, pp. 1933-1940, 2003.

[13] T. Matsuzaki, M. Yashiro, R. Kaizaki et al., "Synergistic antiproliferative effect of mTOR inhibitors in combination with 5-fluorouracil in scirrhous gastric cancer," Cancer Science, vol. 100, no. 12, pp. 2402-2410, 2009.

[14] M. A. Baron, "Structure of intestinal peritoneum in man," American Journal of Anatomy, vol. 69, no. 3, pp. 439-497, 1941.
[15] G. Los, P. H. A. Mutsaers, W. J. F. van der Vijgh, G. S. Baldew, P. W. de Graaf, and J. G. McVie, "Direct diffusion of cisdiamminedichloroplatinum(II) in intraperitoneal rat tumors and intraperitonal chemotherapy: a comparison with systemic chemotherapy," Cancer Research, vol. 49, no. 12, pp. 33803384, 1989.

[16] E. de Bree and D. D. Tsiftsis, "Experimental and pharmacologic studies in intraperitoneal chemotherapy from laboratory bench to bedside," in Advances in Peritoneal Surface Oncology, S. Gonzalez-Moleno, Ed., pp. 53-73, Springer, 2007.

[17] Y. Yonemura, Y. Endou, E. Bando et al., "Effect of intraperitoneal administration of docetaxel on peritoneal dissemination of gastric cancer," Cancer Letters, vol. 210, no. 2, pp. 189196, 2004.

[18] Y. Yonemura, E. Bandou, T. Sawa et al., "Neoadjuvant treatment of gastric cancer with peritoneal dissemination," European Journal of Surgical Oncology, vol. 32, no. 6, pp. 661-665, 2006.

[19] J. L. Koh, T. D. Yan, D. Glenn, and D. L. Morris, "Evaluation of preoperative computed tomography in estimating peritoneal cancer index in colorectal peritoneal carcinomatosis," Annals of Surgical Oncology, vol. 16, no. 2, pp. 327-333, 2009.

[20] M. Inokuchi, T. Yamashita, H. Yamada et al., "Phase I/II study of S-1 combined with irinotecan for metastatic advanced gastric cancer," British Journal of Cancer, vol. 94, no. 8, pp. 1130-1135, 2006.

[21] H. Baba, M. Yamamoto, K. Endo et al., "Clinical efficacy of S-1 combined with cisplatin for advanced gastric cancer," Gastric Cancer, vol. 6, 1, pp. 45-49, 2003.

[22] M. Valle and A. Garofalo, "Laparoscopic staging of peritoneal surface malignancies," European Journal of Surgical Oncology, vol. 32, no. 6, pp. 625-627, 2006.

[23] A. T. Culliford, A. D. Brooks, S. Sharma et al., "Surgical debulking and intraperitoneal chemotherapy for established peritoneal metastases from colon and appendix cancer," Annals of Surgical Oncology, vol. 8, no. 10, pp. 787-795, 2001.

[24] O. Glehen, F. Mithieux, D. Osinsky et al., "Surgery combined with peritonectomy procedures and intraperitoneal chemohyperthermia in abdominal cancers with peritoneal carcinomatosis: a phase II study," Journal of Clinical Oncology, vol. 21, no. 5, pp. 799-806, 2003. 


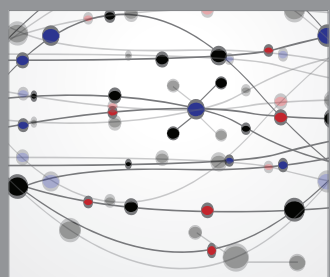

The Scientific World Journal
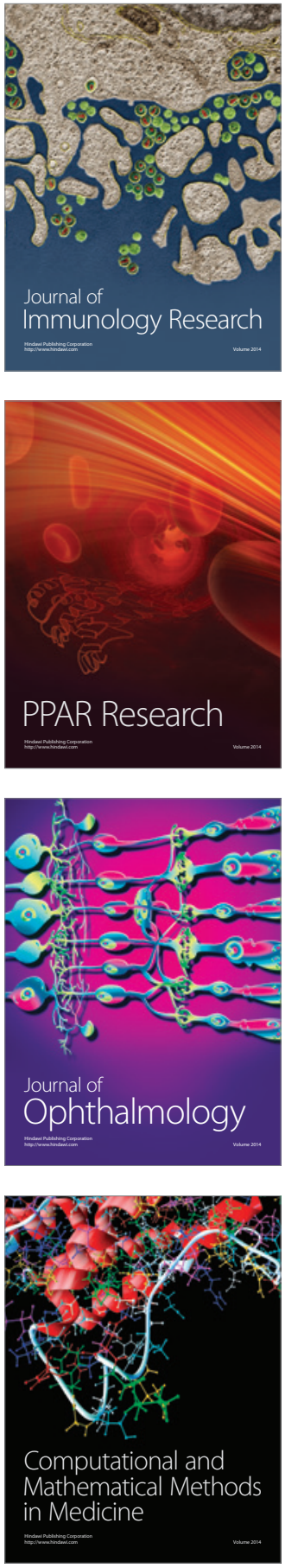

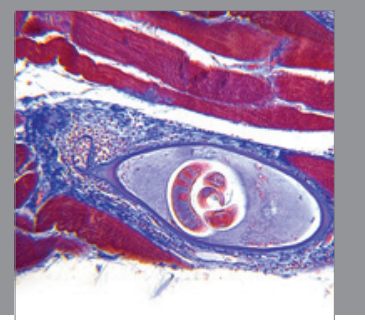

Gastroenterology

Research and Practice
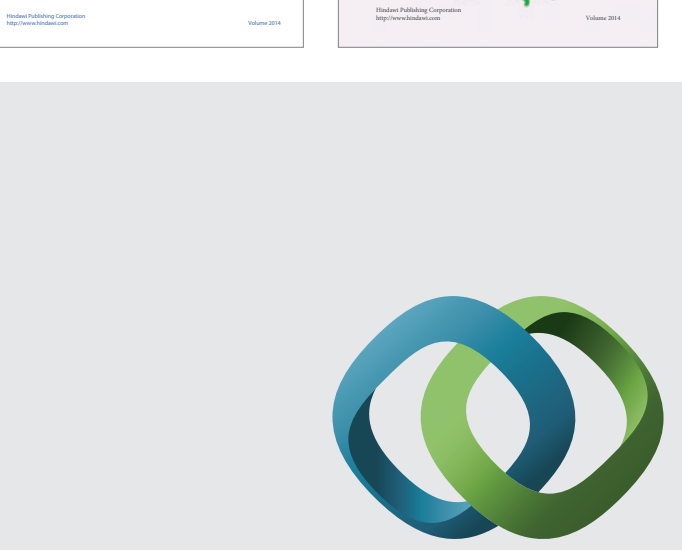

\section{Hindawi}

Submit your manuscripts at

http://www.hindawi.com
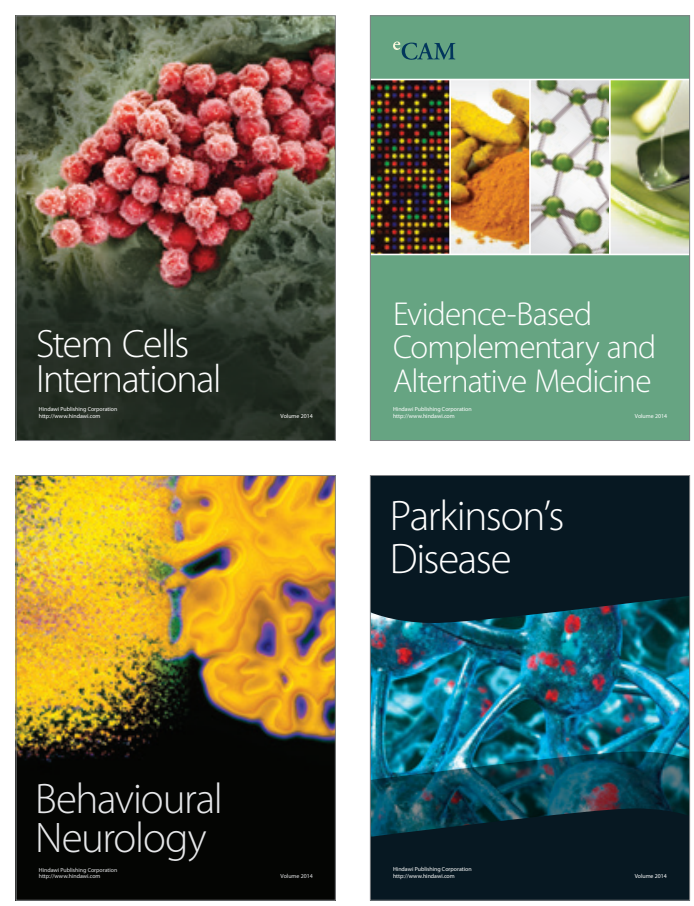

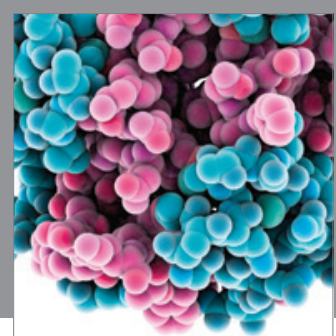

Journal of
Diabetes Research

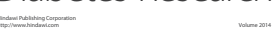

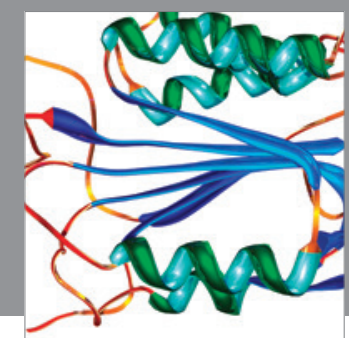

Disease Markers
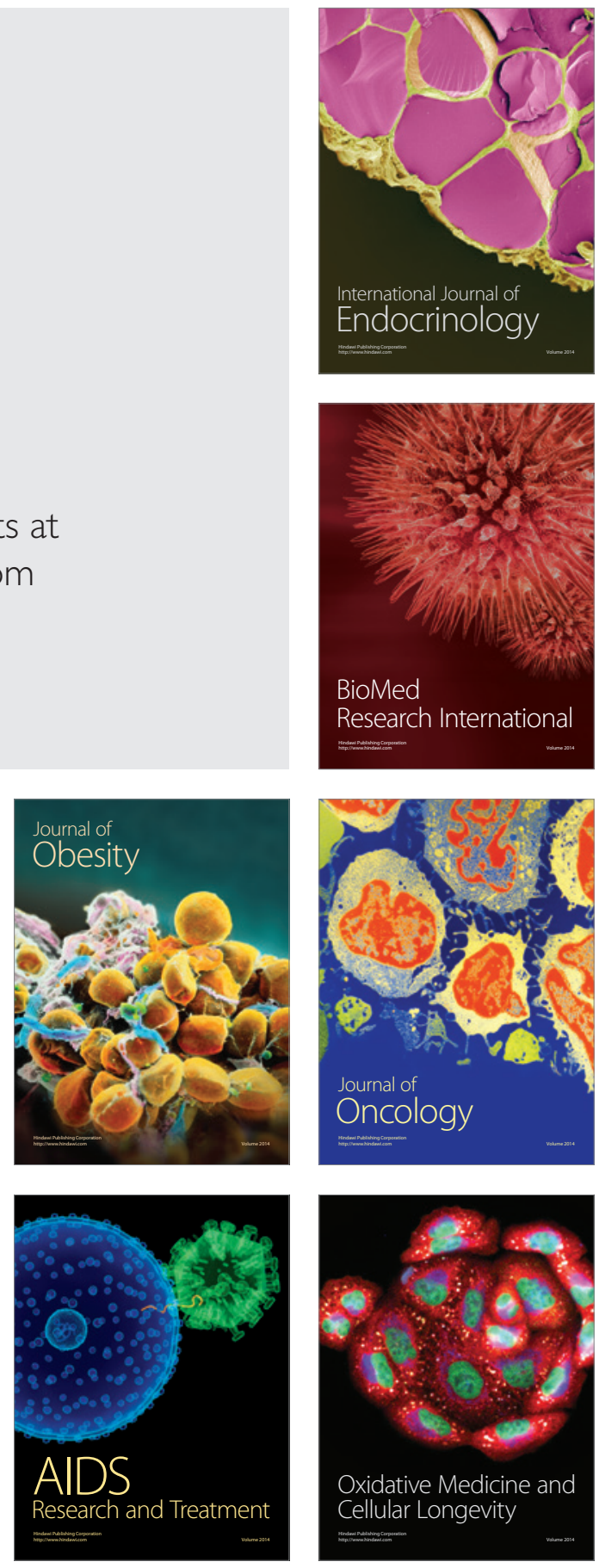